\title{
Design of a Practical and Compact mm-Wave MIMO System with Optimized Capacity and Phased Arrays
}

\author{
Tommaso Cella, ${ }^{1}$ Pål Orten, ${ }^{2}$ and Torbjörn Ekman ${ }^{3}$ \\ ${ }^{1}$ NTNU and UniK, 7491 Trondheim, Norway \\ ${ }^{2} A B B$ and UniK, 1396 Billingstad, Norway \\ ${ }^{3}$ NTNU, 7491 Trondheim, Norway \\ Correspondence should be addressed to Tommaso Cella; tommaso@unik.no
}

Received 21 February 2014; Revised 7 May 2014; Accepted 2 June 2014; Published 24 June 2014

Academic Editor: Joonhyuk Kang

Copyright (C) 2014 Tommaso Cella et al. This is an open access article distributed under the Creative Commons Attribution License, which permits unrestricted use, distribution, and reproduction in any medium, provided the original work is properly cited.

\begin{abstract}
In this paper we evaluate the feasibility of short range outdoor mm-wave MIMO links in the $70 \mathrm{GHz}$ portion of the E-band (71$76 \mathrm{GHz}$ ). We use phased arrays in order to strongly reduce the impact of the multipath components, thus making the channel mainly line-of-sight (LOS). We design the array using a simple patch as a single element and simulate the performances for a $200 \mathrm{~m}$ link and a MIMO system with equal element spacing at the transmitter and the receiver. Each node of the MIMO system consists of a uniform rectangular array (URA) where the single element is a patch antenna, in order to achieve higher gains and narrow beams. Such configuration is much more compact compared to the antennas currently employed for the same bandwidth. We optimize the interelement distances at the transmitter and the receiver and evaluate the capacity achievable with different array sizes. The results show that, for the proposed link budget, capacity up to $29 \mathrm{bit} / \mathrm{s} / \mathrm{Hz}$ is achievable at a range of $200 \mathrm{~m}$, with practical dimensions. We also show that the beamforming capabilities make the design much more flexible than the single reflector antenna systems. In the last part of the paper, we verify that our antenna can also operate in rainy conditions and longer ranges.
\end{abstract}

\section{Introduction}

Research on mm-wave communications has been significantly increased during the last years. Even though mmwave extends from 30 to $300 \mathrm{GHz}$, there are three bands to which we usually refer, which are the V-band $(57-66 \mathrm{GHz})$, the E-band $(71-76 \mathrm{GHz}$ and $81-86 \mathrm{GHz})$, and the W-band $(92-95 \mathrm{GHz})$. The main difference between mm-wave communications compared to lower frequency bands is that they experience a much higher attenuation. This is due to the increased free space propagation loss and the atmospheric absorption. However, while the E-band and the W-band exhibit a reasonable atmospheric absorption, the V-band suffers significant attenuation due to the oxygen absorption [1]. Due to that, the V-band is primarily investigated for indoor communications, while most of the commercially available transceivers intended for outdoor applications are designed in the E-band. Several studies have demonstrated that communications at those bands are mainly LOS [2]. Some possible applications include $4 \mathrm{G} / \mathrm{LTE}$ backhaul, disaster recovery, remote storage access, and fiber extension. The commonly used antenna elements are parabolic reflectors, with very high antenna gains, up to $50 \mathrm{dBi}$. The antenna diameters are in the order of $30-60 \mathrm{~cm}$ with very narrow beams, down to less than $1^{\circ}$ half power beamwidth (HPBW) [3]. The dimensions vary depending on the range and the link budget. Such antennas are very sensitive to positioning, and installing them is not trivial. In addition, the link is fixed and limited to one transmitter and one receiver. One of the main reasons for the increased research in the mm-wave bands is the demand for higher capacity. An attractive method to improve the system capacity is the use of MIMO [4] taking advantage of multiplexing gain as multiple information streams are sent from different transmit antennas towards different receive antennas at the same frequency. In order to get spatial multiplexing at lower frequencies, rich multipath is needed. The 
main advantage of using MIMO at mm-wave bands is that by having a proper inter-element spacing in both transmitting and receiving antennas, multiple independent streams, and thus high capacity, can be obtained, even in LOS [5]. Different LOS MIMO systems have been proposed and tested indoor [6] and outdoor [7, 8]. Another unique characteristic of mmwave band communications is that highly directional beams can be obtained using relatively small antennas. In this paper, we propose a feasibility study of a LOS MIMO radio link aided by high gain antennas implemented using URA. In our design, the transmit and receive antennas consist of multiple uniform rectangular array (URA) [9] with proper spacing to achieve LOS MIMO. The URA provide additional gain through narrow beams. The single antenna element in the URA is a patch. Such antennas are small at those frequencies and cheaper compared to the reflector antennas. In addition, due to the planar shape of the antennas, their placement could be easier. Such combinations of advanced MIMO schemes and practical designs have not been presented by other authors for outdoor scenarios, to our knowledge. Millimeter wave antenna systems have been recently tested indoor [10] and outdoor [11]. We show the possibility of using patch antenna arrays for short range outdoor MIMO links, which provide gain comparable to the commonly used parabolic reflectors, guaranteeing enough received power. In addition, we limit the contribution of the multipath components by using a sufficient number of antenna elements. This reduces the array HPBW, thus obtaining characteristics comparable to a LOS channel. We will show later in this paper that such a configuration is much smaller than using reflector antennas. The antenna would be possible to produce with much lower cost and could be easily located due to the planar shape of such antennas. In addition to the benefits of using MIMO, the system we propose can take advantages of the use of beamforming. The beamforming capabilities of each URA could provide more flexible links, because each array could focus the beam towards receivers placed in different locations, avoiding the time consuming and costly positioning of very narrow beam antennas [12]. Such a system could be used for communications in enterprises, for disaster recovery or fast deployed fiber replacement. We focus our study on the $70 \mathrm{GHz}$ portion of the E-band $(71-76 \mathrm{GHz})$.

In the results section, we will first show the performances achievable for a range up to $200 \mathrm{~m}$. The geometry of the proposed system is shown in Figure 1. We design the array and simulate the mm-wave channel with ray tracing. We then show that high capacity could be obtained for a range longer than $200 \mathrm{~m}$, if particular attention is taken during the array design, even during rainy conditions. The novelty of the proposed system lies in the combination of LOS MIMO communication together with the geometry adopted and shown in Figure 1. This gives a flexible and practical alternative to more costly components. The rest of the paper is organized as follows. In Section 2 the capacity of MIMO systems is described. Section 3 will focus on the MIMO channel model and will introduce the optimal geometry for the system designed in the paper. In Section 4 the characteristics of our system are presented, while simulation

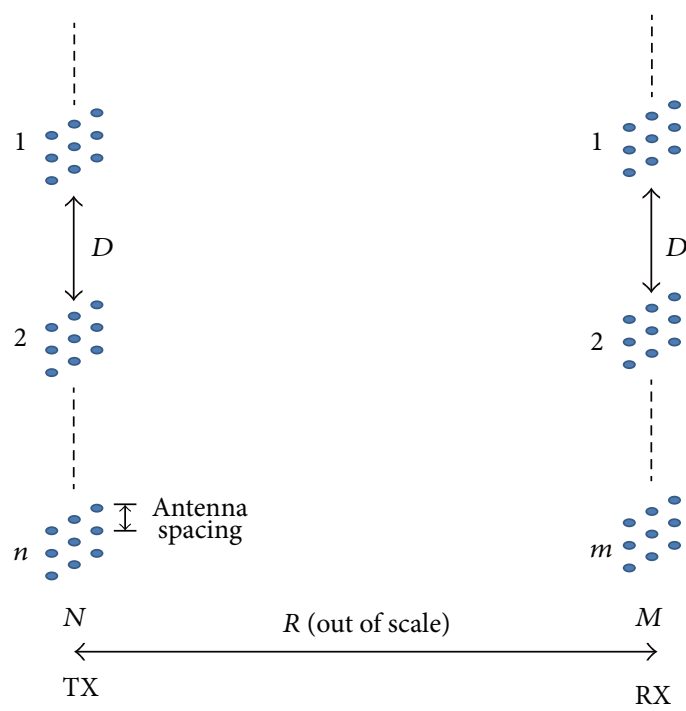

Figure 1: $N \times M$ MIMO system geometry. Each MIMO node is a URA.

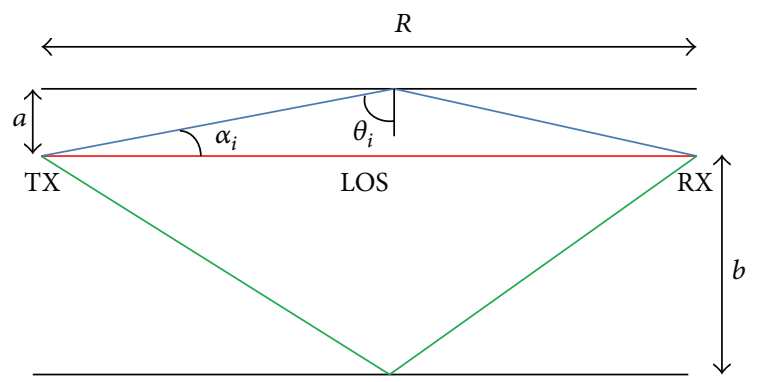

FIGURE 2: Environment (aerial view). The LOS line is normal to the URA plane.

results are shown in Section 5. Finally the paper is concluded in Section 6.

\section{MIMO Capacity}

A MIMO transmission system employs a number of transmit and receive antennas to transmit data over a channel. We denote the number of transmit antennas by $N$ and the number of receive antennas by $M$. Assuming slowly varying and frequency flat fading channels, we can model the MIMO transmission as $\mathbf{r}=\mathbf{H s}+\mathbf{n}$ [13], where $\mathbf{r}$ is the $M \times 1$ received complex-valuated signal vector, $\mathbf{s}$ is the $N \times 1$ transmitted complex-valued signal vector, $\mathbf{H}$ is the $M \times N$ complex-valued channel matrix, and $\mathbf{n}$ is the $M \times 1$ complex-valued additive white Gaussian noise (AWGN) vector with variance $\sigma_{n}^{2}$. We consider uncorrelated branch sources with equal power $P_{T}$ and assume that all receiver antennas experience the same averaged received power $P_{R}$. The average received signal-tonoise ratio (SNR) at one receive antenna becomes $\bar{\gamma}=P_{R} / \sigma_{n}^{2}$. For convenience we define the variables $U=\min (M, N)$ and 
$V=\max (M, N)$ for use in the rest of the paper. With our assumptions, the MIMO capacity can be written as [14]

$$
C=\sum_{i=1}^{U} \log _{2}\left(1+\frac{\bar{\gamma}}{M} \lambda_{i}\right)[\text { bits } / \mathrm{s} / \mathrm{Hz}]
$$

Here, $\lambda_{i}$ is the $i$ th eigenvalue of $\mathbf{W}$, which is defined as

$$
\mathbf{W}= \begin{cases}\mathbf{H H}^{H}, & M \leq N, \\ \mathbf{H}^{H} \mathbf{H}, & M \geq N,\end{cases}
$$

where $\mathbf{H}^{H}$ is the Hermitian transpose of the $\mathbf{H}$ matrix. Equation (1) shows that a MIMO system can be viewed as consisting of $U$ parallel single-input-single-output (SISO) channels, where each channel has gain $\lambda_{i}$ and an average SNR downscaled with the number of transmitters. Because of the characteristics of our system, as will be clear in the next sections, we consider only the LOS channel. Therefore the channel matrix $\mathbf{H}$ is calculated including only the LOS components. For details see [5].

\section{Channel Modeling}

To emulate the mm-wave channel and perform the simulations, we used Wireless Insite [15]. We used the full 3D model which includes the effects of reflections, transmissions, and diffractions on the electric field and the shooting and bouncing ray (SBR) method $[16,17]$. For mm-wave bands, it is common to model the outdoor channel considering only some reflected rays, typically first and second order reflections, because higher order reflected rays will be strongly attenuated, and their contribution will be negligible. It has been demonstrated that, even for a single reflection, the power of a multipath signal is significantly lower than the one of the LOS signal [18]. The distance from the antennas to any reflector has a crucial role in determining the number of rays. In the next section, it will be shown that, because of the range, frequency, and use of subarrays, the channel can be approached as LOS.

LOS Channel: Ray Tracing. It has been demonstrated that, in order to optimize the MIMO capacity in a LOS scenario, the antennas must be properly spaced. This is because a proper positioning of the antennas leads to a high rank LOS channel matrix, corresponding to many nonzero eigenvalues $\lambda_{i}$. The optimal antenna separation with regard to Shannon capacity for a pure LOS channel for ULAs with arbitrary orientation was already found in [5] and is given as

$$
\mathbf{D}=\sqrt{\frac{R \lambda}{M}} .
$$

In (5), $D$ represents the antenna spacing at the transmitter and the receiver, $R$ is the range, and $\lambda$ is the wavelength. $M$ is the number of receivers. We consider the same distance $D$ in this paper. By using (4) in the system design, the optimal solution of (2) is achieved. This is verified when all eigenvalues are equal (i.e., $\lambda_{1}=\lambda_{2}=\cdots=\lambda_{U}=V$ ) and is proven in [5]. In this paper we consider the central point of each URA in order to calculate the antenna distances. It has been demonstrated in [19] that for small variations of the antenna distances there is only a very small decrease in capacity.

\section{System Description}

4.1. Environment Geometry. In this section we describe the geometry for our application, which can be seen in Figure 2. We study a short range outdoor scenario in which the transmitter and receiver are located on two building walls. We consider the case where the LOS path is always present. The range is varying up to $200 \mathrm{~m}$. In Figure 2 we also show the first order reflecting paths between the transmitter and receiver which might be due to different obstacles such as buildings. The vertical distance between the TX and any obstacle and the RX and any obstacle is indicated by $a$ and $b$ in Figure 2 . As explained in Section 3, we consider only the LOS path. The reason for that is shown in Figure 3, where we plot the first order reflection angles at different vertical distances for the reflecting surfaces. As we can see, the first reflection path angle $\left(\alpha_{i}\right)$ is minimum $3^{\circ}$ for an obstacle distance equal to $5 \mathrm{~m}$ for the range of $200 \mathrm{~m}$. Higher order reflections will need even higher angles. The distance from the antennas to any reflector has a crucial role in determining the number of rays. In Figure 4 we plot the angles $\alpha$, which indicate how close an obstacle must be in order for the first reflecting path to be in the receiver antenna pattern. The area below each range line indicates the only LOS area for that specific range. What we can see is that only rays with small direct angles make a strong contribution to the total received power [20]. For our considered narrow beam systems, the reflectors will have to be quite close to the path since we also have short range due to high attenuation. The same considerations are valid for the ground reflection. We model the application scenario with a ray tracing software, Wireless Insite, which was described in the previous section. We will show that the effect of the multipath components can be severely limited by narrowing the antenna beamwidth using phased arrays.

In order to achieve the desired capacity, there are multiple elements at the transmitter and the receiver spaced according to (3). In Table 1 are shown the distances between the MIMO nodes in $\mathrm{m}$ for different range values at the frequency of $73 \mathrm{GHz}$ as well as the array vertical size from the system shown in Figure 1.

4.2. Link Budget. We consider an example system operating in the $70 \mathrm{GHz}$ portion of the E-band $(71-76 \mathrm{GHz})$. The values are shown in Table 2 and can vary depending on the application considered. As the first stage we consider clear sky; therefore there is no rain attenuation. The atmospheric absorption at sea level is about $1 \mathrm{~dB} / \mathrm{Km}$ [21]. We set the transmit power to $15 \mathrm{dBm}$, and the antenna gains are initially fixed to $30 \mathrm{dBi}$. The path loss is calculated for a range equal to $200 \mathrm{~m}$ according to [22]. The noise power in $\mathrm{dBm}$ at the input to a receiver is given by

$$
P_{\text {noise }}=10 \log \left(k T_{\text {syst }}\right)+10 \log (B)+N F_{\mathrm{RX}} \text {, }
$$




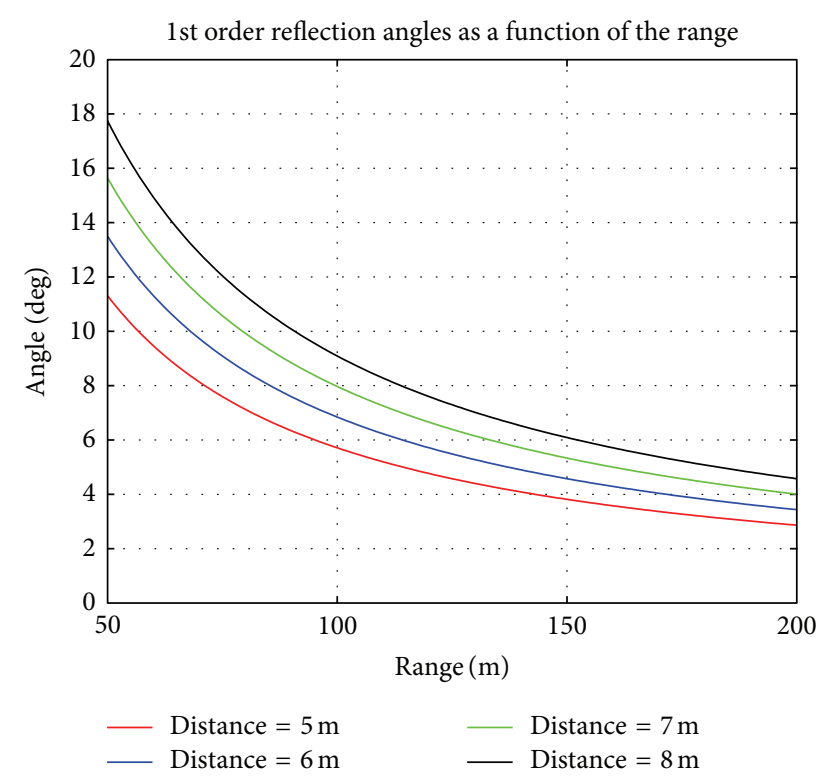

Figure 3: Reflection angles $\left(\alpha_{i}\right)$ for different obstacles distances as a function of the range.

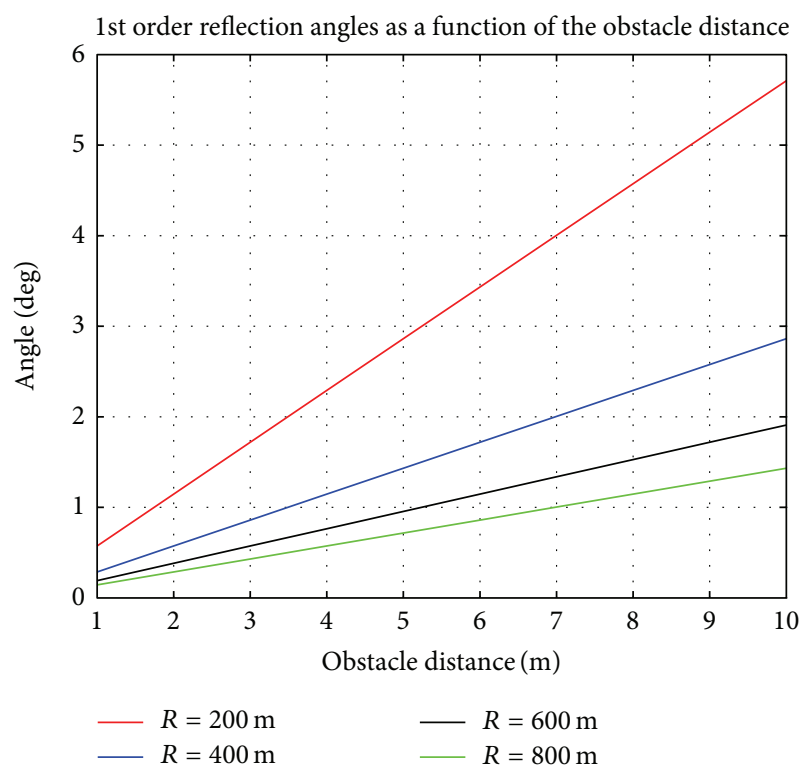

FIGURE 4: First order reflection angles $\alpha$ as a function of the obstacle distance $(a)$.

where $k$ is the Boltzmann's constant, $T_{\text {syst }}$ is system temperature fixed to $17^{\circ}, B$ is the bandwidth set to $1.5 \mathrm{GHz}$, and $N F_{\mathrm{RX}}$ is the receiver noise figure fixed to $5 \mathrm{~dB}$.

4.3. Array Design. As described before, we will simulate the performance achievable with a planar array antenna. The single element is a simple patch antenna, operating in the lower frequency of the E-band $(71-76 \mathrm{GHz})$ designed at the frequency of $73 \mathrm{GHz}$ that gives $\lambda=4 \mathrm{~mm}$ and simulated with CST Microwave Studio. The dimensions are on the order of $1 \mathrm{~mm}$ and shown on Figure 5. We chose a substrate with
TABLE 1: MIMO element distances and array vertical size (in $\mathrm{m}$ ) for the frequency of $73 \mathrm{GHz}$ at different range.

\begin{tabular}{ccccccc}
\hline \multirow{2}{*}{$N \times M$} & \multicolumn{2}{c}{$50 \mathrm{~m}$} & \multicolumn{2}{c}{$100 \mathrm{~m}$} & \multicolumn{2}{c}{$200 \mathrm{~m}$} \\
& Spacing & Array size & Spacing & Array size & Spacing & Array size \\
\hline $2 \times 2$ & 0,32 & 0,32 & 0,45 & 0,45 & 0,64 & 0,64 \\
$3 \times 3$ & 0,26 & 0,52 & 0,37 & 0,74 & 0,52 & 1,05 \\
$4 \times 4$ & 0,23 & 0,68 & 0,32 & 0,96 & 0,45 & 1,36 \\
$5 \times 5$ & 0,20 & 0,81 & 0,29 & 1,15 & 0,40 & 1,62 \\
\hline
\end{tabular}

TABLE 2: Example link budget.

\begin{tabular}{lcc}
\hline Tx power & 15,00 & $\mathrm{dBm}$ \\
GTx & 30,00 & $\mathrm{dBi}$ \\
GRx & 30,00 & $\mathrm{dBi}$ \\
Path loss $(200 \mathrm{~m})$ & $-115,74$ & $\mathrm{~dB}$ \\
Background noise & $-174,00$ & $\mathrm{dBm} / \mathrm{Hz}$ \\
Noise BW & 91,76 & $\mathrm{~dB}$ \\
Noise figure Rx & 5,00 & $\mathrm{~dB}$ \\
Atmospheric absorption & 0,20 & $\mathrm{~dB}$ \\
Rain attenuation & 0,00 & $\mathrm{~dB}$ \\
\hline SNR Rx & 36,30 & $\mathrm{~dB}$ \\
\hline
\end{tabular}

high relative permittivity equal to 10.2 and thickness equal to $75 \mu \mathrm{m}$. The patch gain is $6.8 \mathrm{dBi}$ and the polarization is vertical. In order to achieve the desired beamwidth and gain, we simulated different array sizes. In Figure 5 we show the radiation pattern for the single element, as well as $16 \times 16$ and $32 \times 32$ arrays. The maximum element spacing for a chosen maximum scan angle to avoid grating lobes is given by [23]

$$
d_{\max }=\frac{\lambda}{1+\sin (\alpha)},
$$

where $\lambda$ is the wavelength and $\alpha$ is the scan angle. We consider scan angles up to $\pm 30^{\circ}$ from broadside, which gives a maximum antenna spacing equal to $2 / 3 \lambda$. We set it to be $0.6 \lambda$ in order to reduce the HPBW achievable with the typical $0.5 \lambda$ spacing. The array lengths are then, respectively, $36,9 \mathrm{~mm}$ and $76,3 \mathrm{~mm}$. Such dimensions are quite small compared to the reflector antennas typically used outdoor in the E-band. In Figure 5 we also show the HPBW and side lobe level (SLL) for the $16 \times 16$ and $32 \times 32$ array. As we can see, the maximum gain is, respectively, $30.7 \mathrm{dBi}$ and $37.9 \mathrm{dBi}$, while the HPBW is $5.2^{\circ}$ and $2.4^{\circ}$ for uniform excitation.

\section{Results}

In this section we show the simulation results. We simulate the achievable capacity for a $2 \times 2$ MIMO system. The capacity was simulated with MATLAB. Due to the constructive and destructive effect of the multipath components, the received power can vary significantly. By choosing narrow antenna beams we want to limit their contribution. In Figure 6 we plot the received power at the range of $200 \mathrm{~m}$ by varying the reflector vertical distance, which we set as wall, from 0 to $15 \mathrm{~m}$ 


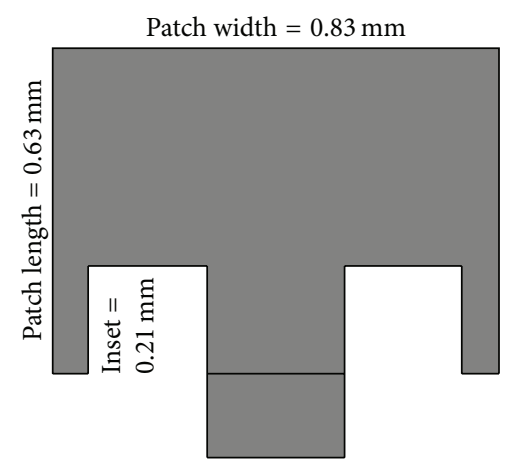

(a)

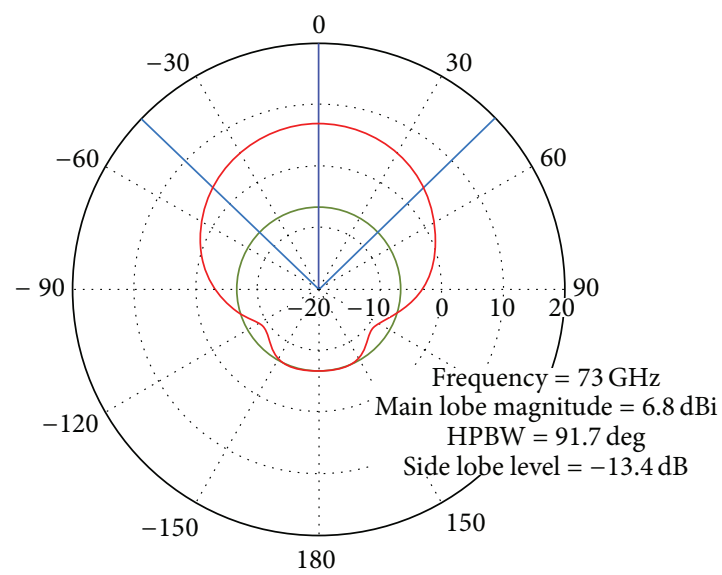

(c)

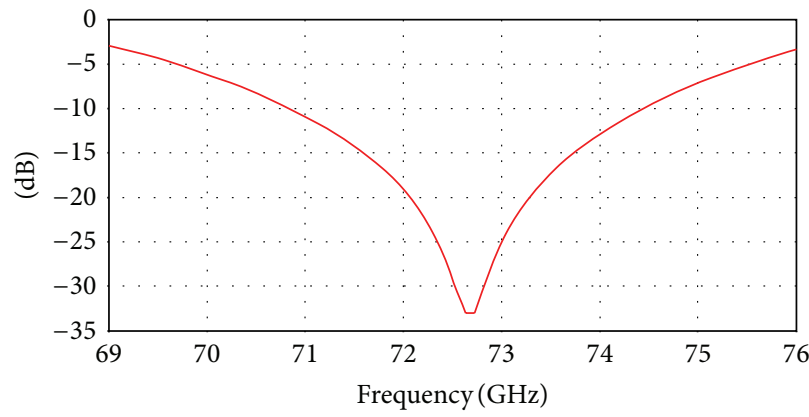

(b)

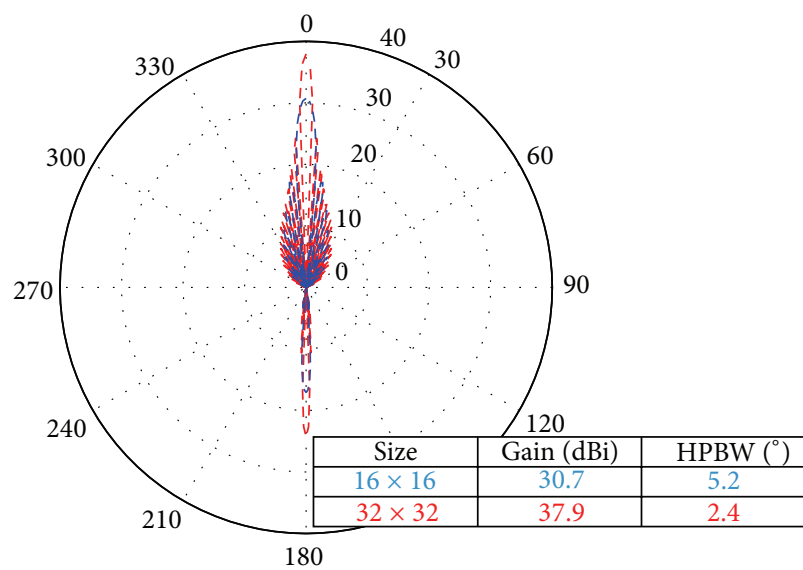

- - - Array $16 \times 16$

- - - Array $32 \times 32$

(d)

Figure 5: (a) Designed patch antenna, (b) reflection coefficient, (c) single element E-field, (d) $16 \times 16$ and $32 \times 32$ array E-field.

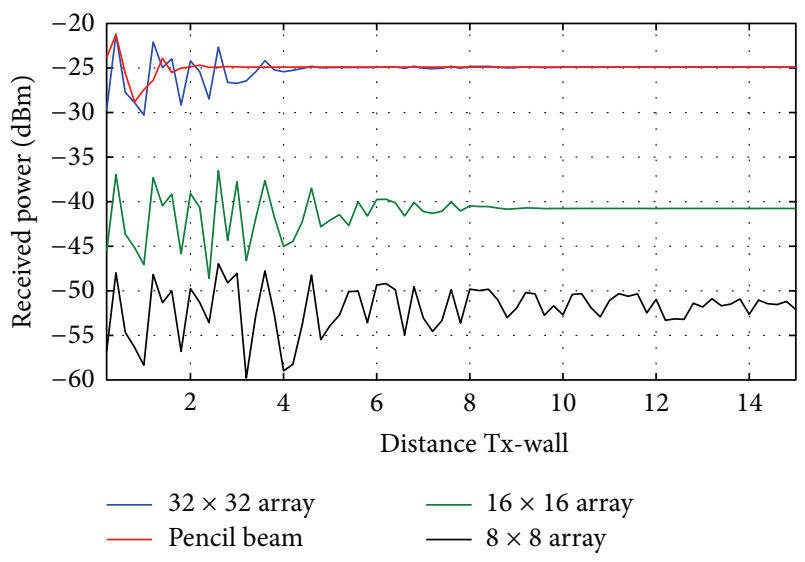

FIGURE 6: Received power for different wall distances.

(a and $b$ in Figure 2 ) for the $16 \times 16$ and $32 \times 32$ arrays. The wall material is concrete and we consider vertical polarization. In Figure 6 we also included the received power of a pencil beam pattern with the same gain and HPBW equal to $1^{\circ}$, which is comparable to the reflector antennas used at those frequencies, as well as an $8 \times 8$ element array. This shows that the more we increase the array size, the more the channel will result in LOS. As we can see, if the reflectors, which we assume to be walls in this case, are not closer than $5 \mathrm{~m}$ from the LOS path, we can consider the channel a pure LOS for the $32 \times 32$ element array case. For the pencil beam antenna, the channel is LOS even if obstacles are very close, as expected. Therefore for our capacity simulations we consider the $32 \times 32$ array as a MIMO node. As described before, we do not include the ground reflection in our model. The reason for this choice is clear from Figure 7. We place both the transmitter and the receiver at different heights from 1 to $10 \mathrm{~m}$, and we plot the difference between the LOS and the ground reflection component for different materials. As expected, the ground reflection impact is comparable to the first order reflection path; in fact for the $32 \times 32$ array the ground reflection is lower than $30 \mathrm{~dB}$ of the LOS path from a height of about $5 \mathrm{~m}$.

One of the advantages of using antenna arrays compared to reflector antennas is that we can use them as phased arrays and point the beam in different directions. In Figure 8 we plot the received power by focusing the beam from broadside to $+30^{\circ}$ in azimuth for the $32 \times 32$ array and $R=200 \mathrm{~m}$. For the calculations we consider no reflectors close to the 


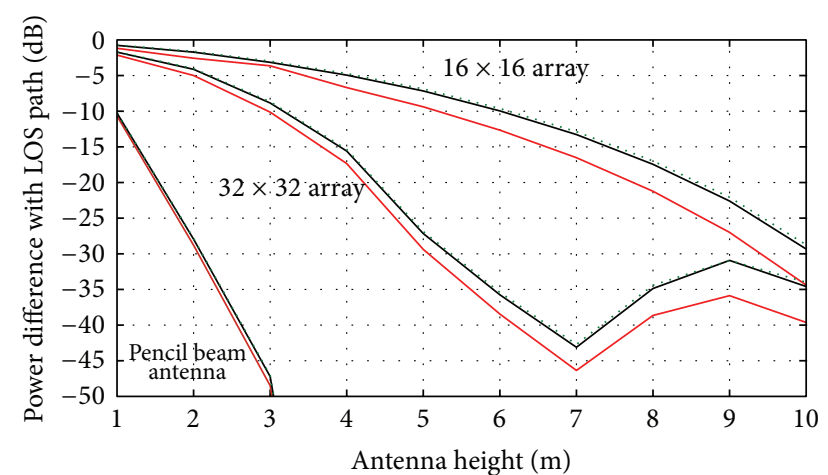

$\begin{array}{ll}\text { _.... Asphalt } & \text { Dry earth } \\ \ldots & \text { Wet earth }\end{array}$

FIGURE 7: LOS-ground reflection power difference for different ground materials.

LOS path, therefore the impact of the multipath components is strongly limited. In Figure 8 the white rectangles represent different buildings from the top and therefore possible receiver locations. Beamforming can be used to point the beams towards receivers at different location electronically. The received power decreases because of the increased range and decreased gain compared to the broadside orientation. As we can see, we can have quite high received power, which reduces by about $5.5 \mathrm{~dB}$ from broadside to $30^{\circ}$ orientation. The HPBW is $2.4^{\circ}$ for the broadside direction and increases up to $2.8^{\circ}$, which means that the assumption of only LOS still remains valid if there are no close reflectors to the TX-RX path. Wider focusing angles are not considered because of the beam broadening and the much higher possibility of strong reflections.

Due to the small wavelength, a proper positioning of the antennas is very hard, and errors are always present. Even a small misalignment can cause the signal level to drop significantly. For our system this can be compensated with adaptive beamforming, while it will require frequent adjustment using single reflector antennas.

In Figure 9, we plot the achievable capacity considering the distances between the arrays optimized for the range equal to $200 \mathrm{~m}$. We use the ergodic capacity in order to keep the result general. Using other performance measures such as error rate would require us to choose a modulation and coding scheme, making the result more specific. The parameters used for the array geometry are calculated according to (3), which has been demonstrated to optimize the MIMO capacity in LOS [5]. We also plot the achievable capacity optimized for each distance, which gives the maximum capacity achievable as upper bound. For all the capacity simulations, we divide the total transmit power of $15 \mathrm{dBm}$ by the number of transmitters. As we can see, the capacity is strongly dependent on the MIMO node distances. In Figure 9 we also plot the achievable capacity for a smaller subarray distance compared to the optimal distance at the range of $200 \mathrm{~m}$, which is $64 \mathrm{~cm}$.
We consider, respectively, $50 \%$ and $75 \%$ of the optimal distance for the range of $200 \mathrm{~m}$, which correspond, respectively, to $32 \mathrm{~cm}$ and $48 \mathrm{~cm}$. As we can see, the result shows that the achievable capacity is lower compared to the optimized dimension, as expected. Still, it could be practical to have smaller arrays; therefore this should be taken into account.

We then wanted to check the possibility of having smaller size subarrays by increasing the number of the MIMO elements. As explained in Section 3, for optimized subarray distances, all the eigenvalues $\lambda_{i}$ of (1) are equal to $V=$ $\max (M, N)$; therefore the capacity as (1) can be expressed by

$$
C=\log _{2}\left(1+\bar{\gamma}_{M}\right) \cdot M[\mathrm{bits} / \mathrm{s} / \mathrm{Hz}]
$$

where $\bar{\gamma}_{M}$ is the average received SNR for $M$ receivers. If we fix $M$, then the only parameters which can change SNR in the link budget are the antenna gains (we still consider equal power transmission). We made the calculations for the range equal to $200 \mathrm{~m}$, where the achievable capacity for a $2 \times 2$ system is equal to $29 \mathrm{bits} / \mathrm{s} / \mathrm{Hz}$. We set this value as reference and calculated how much we could reduce the antenna gain at each node in order to get the same capacity at the distance of $200 \mathrm{~m}$. The results are shown in Table 3. As we can see, by increasing the number of MIMO nodes, a lower array gain is needed. This difference in gain decreases with increasing the number of MIMO nodes. We also show in Table 3 the subarray size reduction for the different systems, as simulated by CST Microwave Studio, while keeping the $2 \times 2$ system as a reference. If we compare the subarray size for $3 \times 3$ and $4 \times 4$ system, we realize that, in order to get the same capacity for the range of $200 \mathrm{~m}$, we could use, respectively, $16 \times 16$ and $11 \times 11$ subarrays, which correspond, respectively, to $50 \%$ and $23.6 \%$ of the total antennas for the $2 \times 2$ system. Anyway, the contributions from reflections are more likely with smaller subarrays as the HPBW increases. Using $200 \mathrm{~m}$ as a reference distance, the achievable capacity for different MIMO constellations is plotted in Figure 10. Moving up from $2 \times 2$ to higher order MIMO, the size of each subarray is reduced according to Table 3 . As we can see, for the reference distance of $200 \mathrm{~m}$, the achievable capacity is the same for all the constellations. We can also notice that, beyond the reference range of $200 \mathrm{~m}$, the capacity for all the MIMO systems is inversely proportional to the number of MIMO elements. We simulated the capacity for a bigger range to have confirmations of that. This tradeoff might also be considered during the design.

The received $\mathrm{S} / \mathrm{N}$ obtained in Table 2 does not take into account the rain attenuation, which has a relevant impact on mm-wave outdoor communications. In Figure 11 we show the $\mathrm{S} / \mathrm{N}$ for different rain conditions for a range up to $1 \mathrm{~km}$ with LOS assumption for the $32 \times 32$ element array and a $2 \times 2$ system. The rain attenuation factors are taken from [24]. We consider antenna gain at the transmitter and receiver equal to $37.9 \mathrm{~dB}$, while the other parameters are taken from Table 2 . The free space loss is calculated according to [22]. As we can see, the $\mathrm{S} / \mathrm{N}$ is strongly influenced by rain, depending on the intensity and the range considered, but reliable communications could be maintained even with heavy rain conditions, as long as sufficient link margin is considered. 


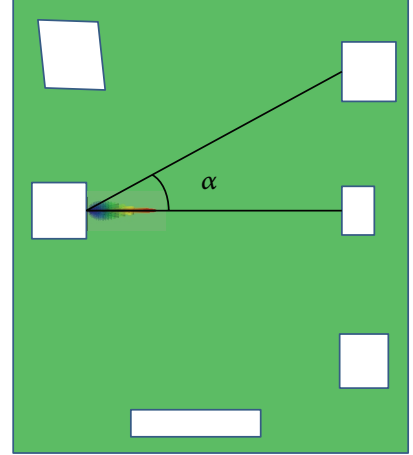

(a)

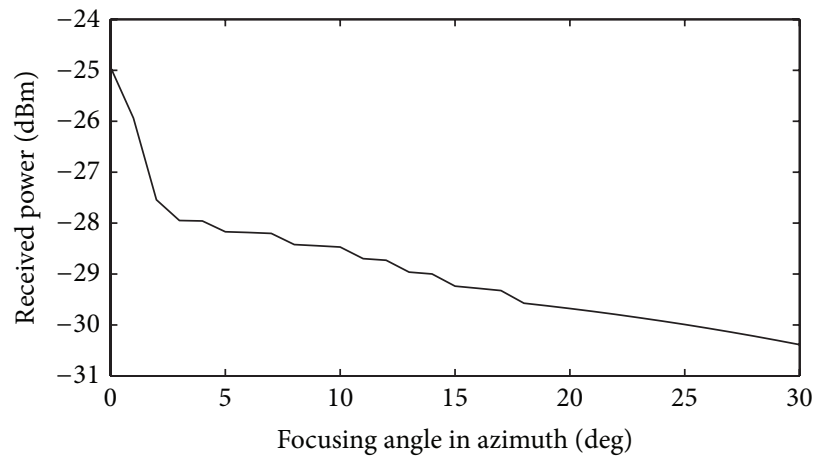

(b)

Figure 8: (a) 2D scenario and (b) received power for different focusing angles (broadside $R=200 \mathrm{~m}$ ).

TABLE 3: Array gain reduction by increasing the number of MIMO nodes $(2 \times 2$ set as reference).

\begin{tabular}{|c|c|c|c|c|}
\hline MIMO elements & Gain difference $(\mathrm{dB})$ & Subarray dimension & Total antennas & Array HPBW $\left(^{\circ}\right)$ \\
\hline $2 \times 2$ & 0 & $32 \times 32$ & 4096 & 2.4 \\
\hline $3 \times 3$ & -7.47 & $16 \times 16$ & 2048 & 5.2 \\
\hline $4 \times 4$ & -11.21 & $11 \times 11$ & 968 & 7.7 \\
\hline $5 \times 5$ & -13.48 & $8 \times 8$ & 576 & 10.6 \\
\hline $6 \times 6$ & -15.00 & $7 \times 7$ & 686 & 12.1 \\
\hline $7 \times 7$ & -16.12 & $6 \times 6$ & 576 & 14.2 \\
\hline $8 \times 8$ & -16.98 & $6 \times 6$ & 576 & 14.2 \\
\hline
\end{tabular}

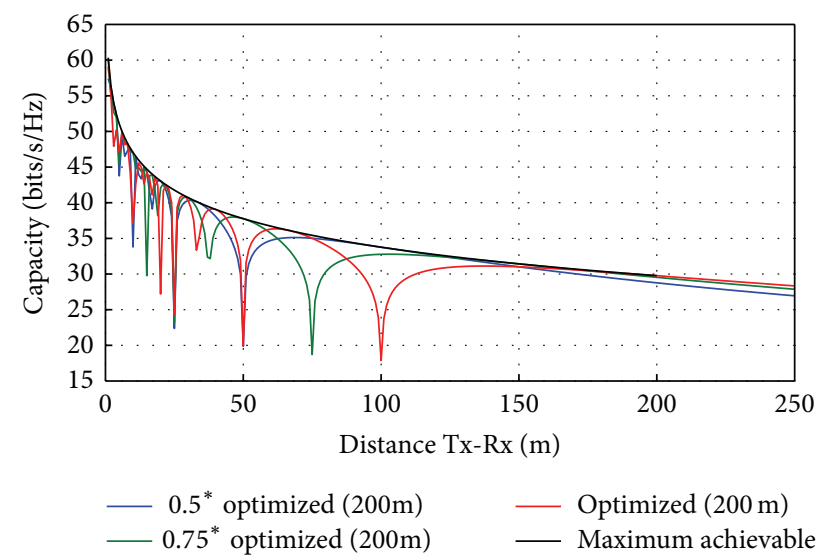

FIGURE 9: Achievable capacity for a $2 \times 2$ MIMO system with different element distances.

\section{Conclusion}

In this paper we study the feasibility of a short range mmwave MIMO LOS radio link outdoor. The system is aided by high gain antennas implemented using URA. We use patch antenna arrays and demonstrate that having such antennas is much more flexible than using the reflector antennas which are commonly used. This is because we take advantage of beamforming. In addition such arrays would be easier to

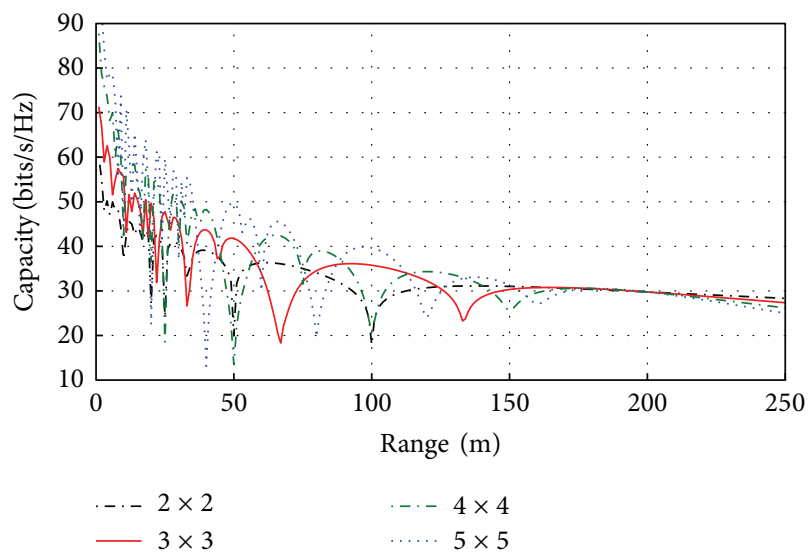

Figure 10: Achievable capacity for $2 \times 2,3 \times 3,4 \times 4$, and $5 \times 5$ MIMO with reduced subarray dimension.

install and cheaper to produce. We first showed that, at a range up to $200 \mathrm{~m}$, the effect of the multipath components could be severely reduced, which will make the channel virtually LOS. We then calculate the achievable capacity for a MIMO system, where each MIMO node is a URA with $32 \times$ 32 antenna elements. The capacity is maximized by a proper spacing between the MIMO elements at the transmitter and receiver. The results show that the use of our configuration 


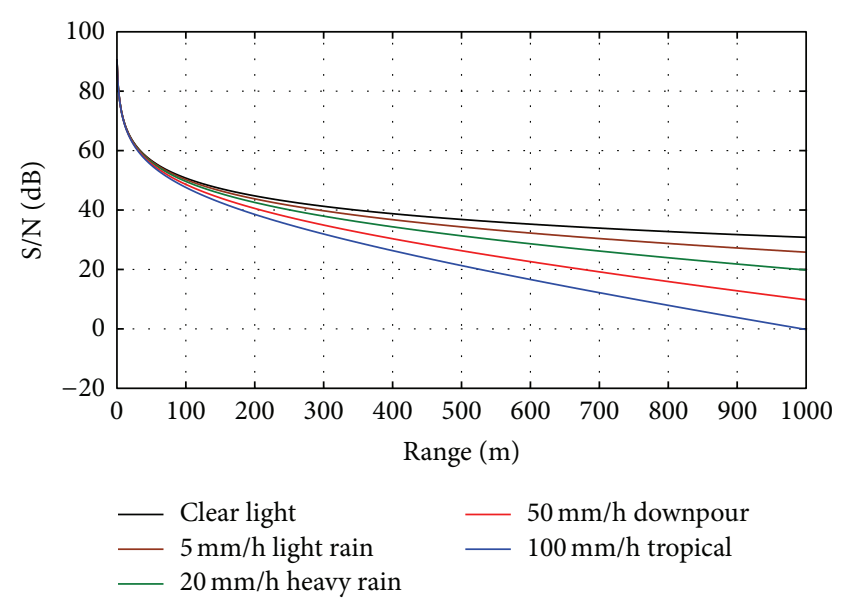

FIGURE 11: Link S/N versus distance for different rain conditions (32 $\times 32$ array).

is able to optimize the capacity for the specific geometry. The results show also that the achievable capacity is still high even for nonoptimal configurations. Furthermore, we demonstrate that, at a specific range, the capacity can be improved with a smaller subarray size, by increasing the number of MIMO elements. In the end we demonstrate that the link could be robust to different rain conditions, if the array is properly designed.

\section{Conflict of Interests}

The authors declare that there is no conflict of interests regarding the publication of this paper.

\section{References}

[1] M. Luise, F. Giannetti, and R. Reggiannini, "Mobile and personal communications in the $60 \mathrm{GHz}$ band: a survey," Wireless Personal Communications, vol. 10, no. 2, pp. 207-243, 1999.

[2] I. Sarris and A. Nix, "Design and performance assessment of maximum capacity MIMO architectures in line-of-sight," IEE Proceedings: Communications, vol. 153, no. 4, pp. 482-488, 2006.

[3] M. Lucente, C. Stallo, T. Rossi et al., "Analysis and design of a point-to-point radio-link at $\mathrm{W}$ band for future satellite telecommunication experiments," in Proceedings of the IEEE Aerospace Conference (AERO '11), Big Sky, March 2011.

[4] D. Jie, J. Wang, H. Zhang, and G. Wang, "Channel capacity of $60 \mathrm{GHz}$ wireless communication systems over indoor lineof-sight and non-line-of-sight channels," in Proceedings of the 6th International Conference on Wireless Communications, Networking and Mobile Computing (WiCOM '10), pp. 1-4, Chengdu, China, September 2010.

[5] F. Bøhagen, P. Orten, and G. E. Øien, "Design of optimal high-rank line-of-sight MIMO channels," IEEE Transactions on Wireless Communications, vol. 6, no. 4, pp. 1420-1424, 2007.

[6] C. Sheldon, E. Torkildson, S. Munkyo, C. P. Yue, U. Madhow, and M. Rodwell, "A $60 \mathrm{GHz}$ line-of-sight $2 \times 2$ MIMO link operating at $1.2 \mathrm{Gbps}$," in Proceedings of the IEEE Antennas and Propagation Society International Symposium (AP-S '08), pp. 14, IEEE, San Diego, Calif, USA, July 2008.
[7] H. Zhang, S. Venkateswaran, and U. Madhow, "Channel modeling and MIMO capacity for outdoor millimeter wave links," in IProceedings of the EEE Wireless Communications and Networking Conference (WCNC '10), Sydney, Australia, April 2010.

[8] C. Sacchi, C. Stallo, and T. Rossi, "Space and frequency multiplexing for MM-wave multi-gigabit point-to-point transmission links," in Proceedings of the IEEE Aerospace Conference (AERO '13), Big Sky, Mont, USA, March 2013.

[9] T. Cella, P. Orten, and T. Ekman, "Wireless access using MIMO subarrays at mm-wave bands," in Proceedings of the IEEE International Symposium on Wireless Pervasive Computing (ISWPC '13), pp. 1-5, Taipei, Taiwan, 2013.

[10] S. Nie, G. R. MacCartney Jr., S. Sun, and T. S. Rappaport, " $72 \mathrm{GHz}$ millimeter wave indoor measurements for wireless and backhaul communications," in Proceedings of the 24th IEEE International Symposium on Personal, Indoor and Mobile Radio Communications: Mobile and Wireless Networks (PIMRC '13), 2013.

[11] T. S. Rappaport, S. Sun, R. Mayzus et al., Millimeter Wave Mobile Communications for $5 G$ Cellular: It Will Work!, IEEE Access, 2013.

[12] T. Cella, P. Orten, and T. Ekman, "Mm-wave short range outdoor links with phased arrays," in Proceedings of the 6th International Symposium on Communications, Control , and Signal Processing (ISCCSP '14)., 2014.

[13] D. Gesbert, M. Shafi, D. S. Shiu, P. J. Smith, and A. Naguib, "From theory to practice: an overview of MIMO space-time coded wireless systems," IEEE Journal on Selected Areas in Communications, vol. 21, no. 3, pp. 281-302, 2003.

[14] E. Telatar, "Capacity of multiantenna gaussian channels," Technical Memorandum, AT\&T Bell Labratories, 1995.

[15] Wireless Insite, http://www.remcom.com/wireless-insite.

[16] J. Schuster and R. Luebbers, "Hybrid SBR/GTD radio propagation model for site-specific predictions in an urban environment," in Proceedings of the 12th Annual Review of Progress in Applied Computational Electromagnetics, vol. 1, pp. 84-92, Monterey, Calif, USA, March 1996.

[17] J. Schuster and R. Luebbers, "Comparison of site-specific radio propagation path loss predictions to measurements in an Urban area," in Proceedings of the IEEE AP-S International Symposium and URSI Radio Science Meeting, vol. 1, pp. 1210-1213, July 1996.

[18] C. Stallo, E. Cianca, S. Mukherjee, T. Rossi, M. de Sanctis, and M. Ruggieri, "UWB for multi-gigabit/s communications beyond $60 \mathrm{GHz}$," Telecommunication Systems, vol. 52, no. 1, pp. 161-181, 2013.

[19] X. Gong, Z. Yuan, J. Xu, and L. Hu, "Light-of-sight MIMO for next-generation microwave transmission systems," ZTE Communications, vol. 10, no. 4, pp. 33-38, 2012.

[20] H. Zhang, S. Venkateswaran, and U. Madhow, "Channel modeling and MIMO capacity for outdoor millimeter wave links," in Proceedings of the IEEE Wireless Communications and Networking Conference (WCNC '10), Sydney, Australia, April 2010.

[21] F.T. Ulaby and A. Straiton, "Atmospheric absorption of radio waves between 150 and $350 \mathrm{GHz}$," IEEE Transactions on Antennas and Propagation, vol. 18, no. 4, pp. 479-485, 1970.

[22] ITU, "Propagation data and prediction methods for the planning of indoor radiocommunication systems and radio local area networks in the frequency range $900 \mathrm{MHz}$ to $100 \mathrm{GHz}$," Recommendation ITU-R P.1238-7, 2012. 
[23] R. C. Johnson, H. Jasik, and H. B. Crawford, Antenna Engineering Handbook, McGraw Hill, 2nd edition, 1999.

[24] ETSI TS 102 524, "Fixed Radio Systems; Point-to-Point equipment; Radio equipment and antennas for use in Point-to-Point Millimetre wave applications in the Fixed Services ( mmwFS ) frequency bands $71 \mathrm{GHz}$ to $76 \mathrm{GHz}$ and $81 \mathrm{GHz}$ to $86 \mathrm{GHz}$," July 2006. 

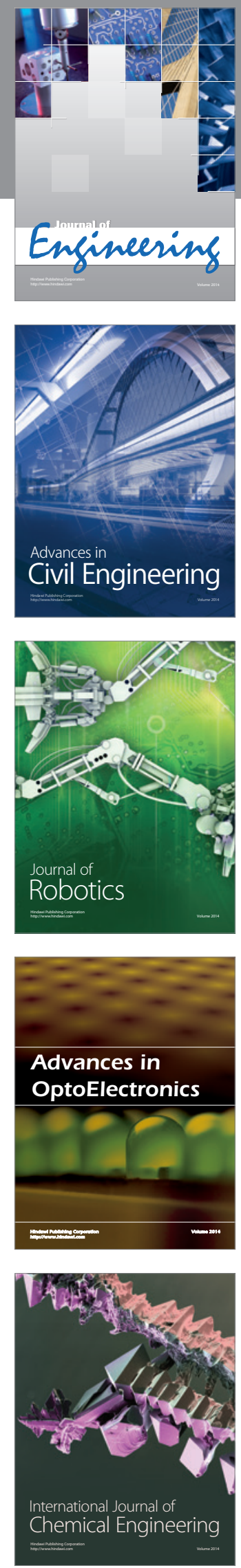

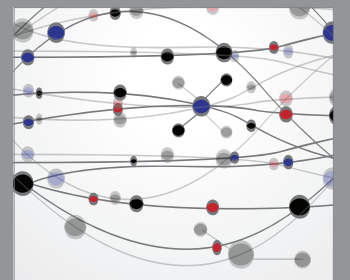

The Scientific World Journal
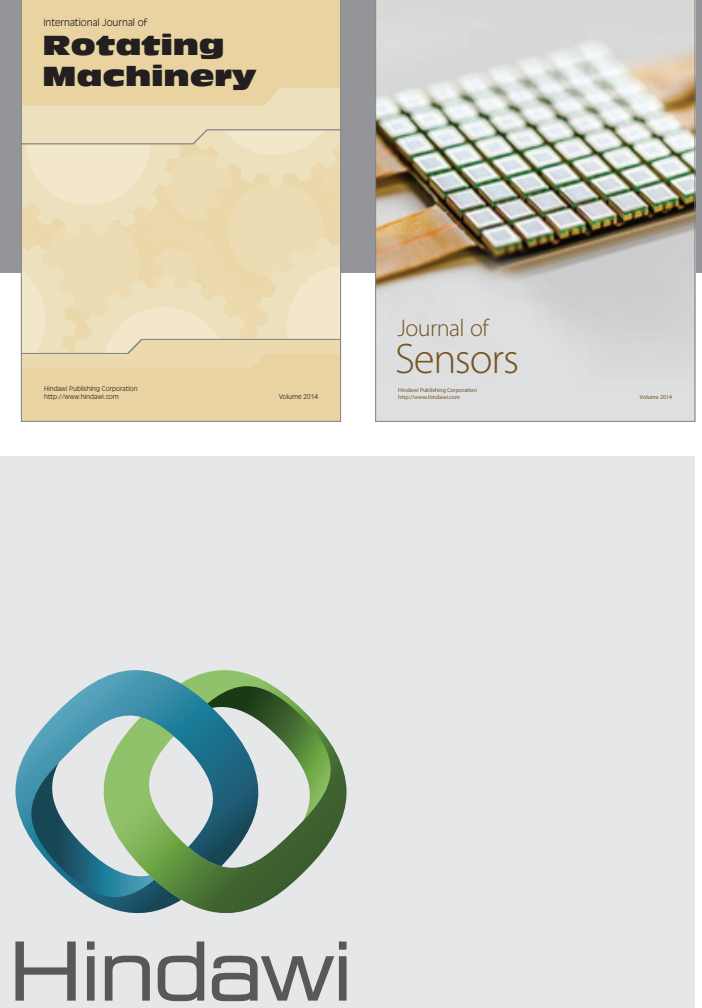

Submit your manuscripts at http://www.hindawi.com
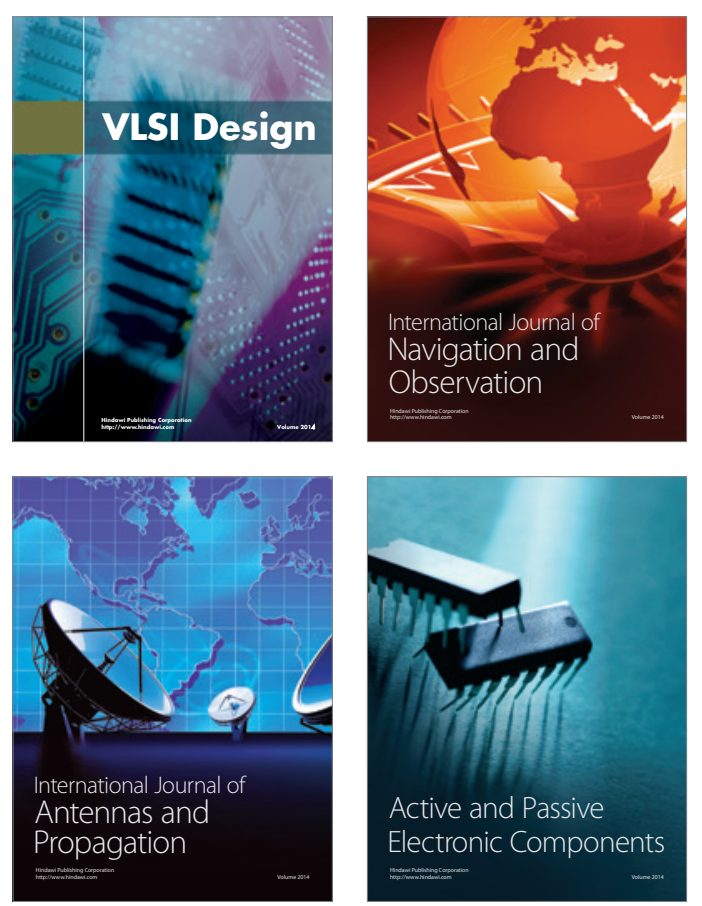
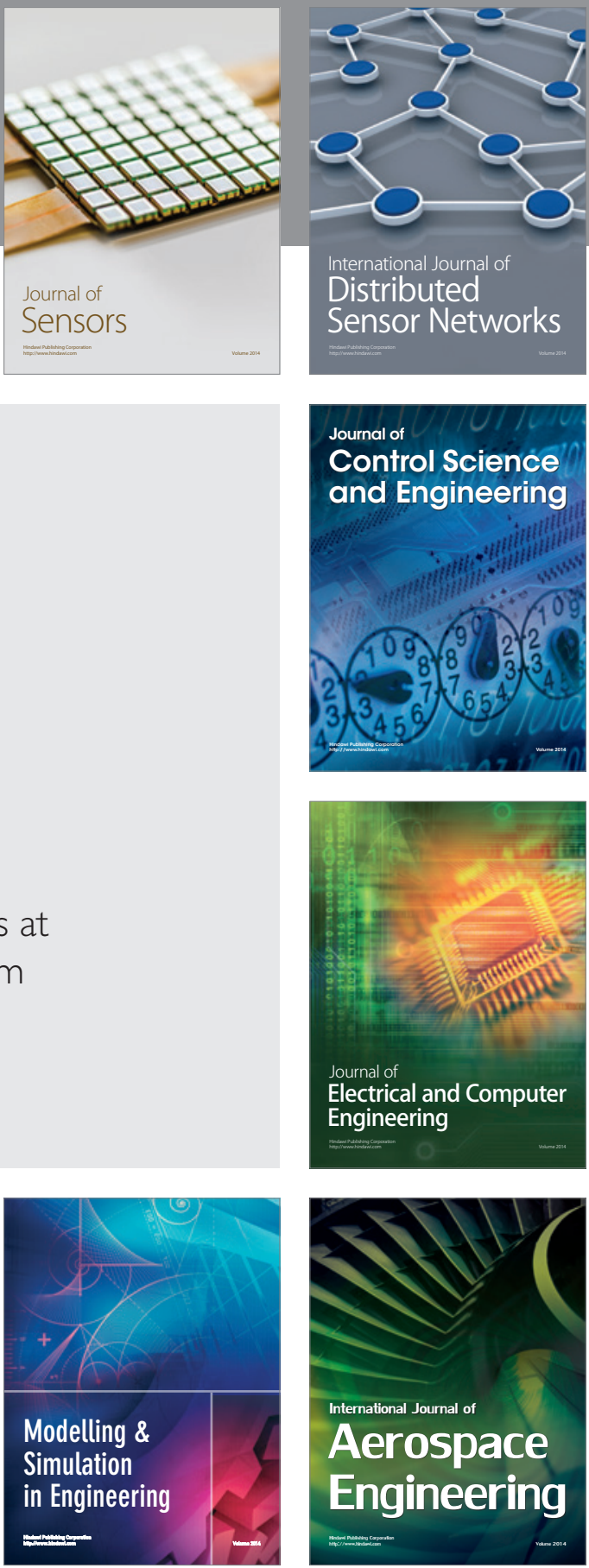

Journal of

Control Science

and Engineering
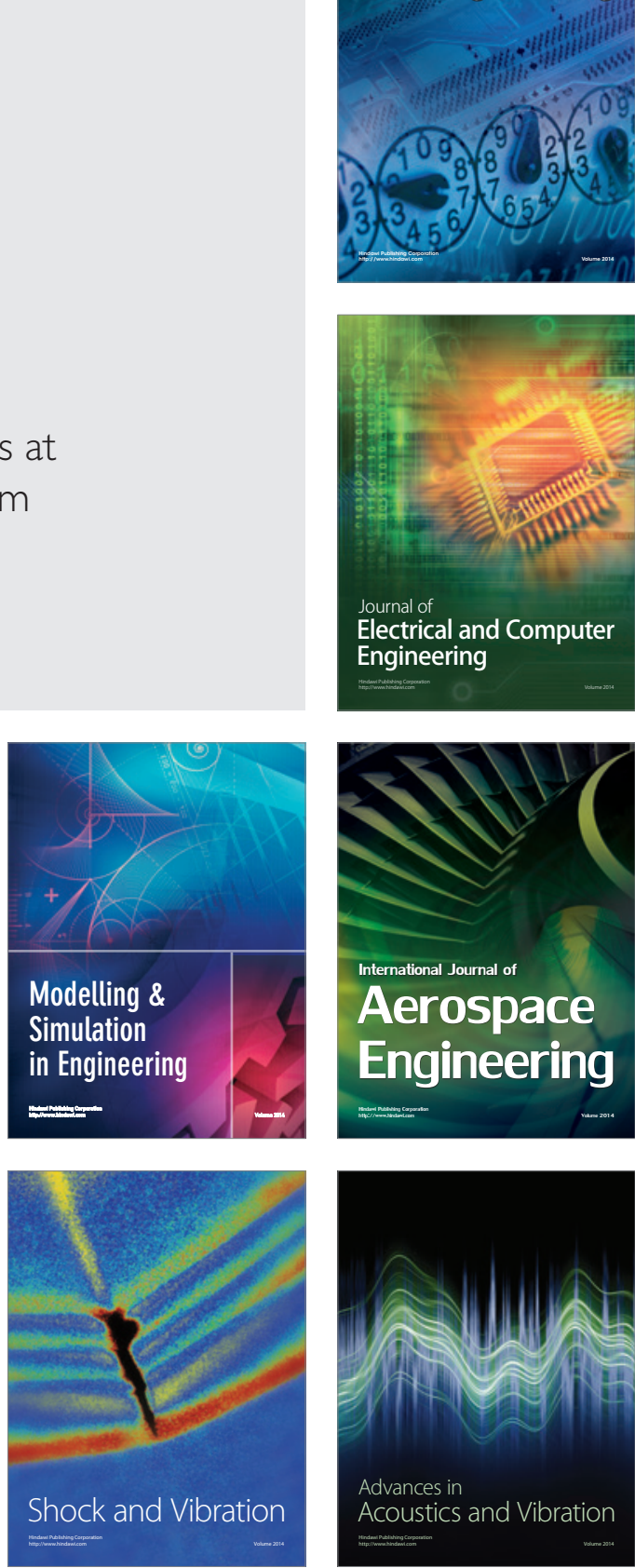